\title{
Maintenance Therapy with Rituximab in Adult Patients with Immune Thrombocytopenia
}

\author{
Marica Pavkovic, Slobodanka Trpkovska-Terzieva, Tatjana Sotirova, Lidija Cevreska, Aleksandar Stojanovic \\ University Clinic for Hematology, Medical Faculty, Ss Cyril and Methodius University Skopje, Republic of Macedonia
}

\author{
Citation: Pavkovic M, Trpkovska-Terzieva S, \\ Sotirova T, CevreskaL, Stojanovic A. Maintenance \\ Therapy with Rituximab in Adult Patients with \\ Immune Thrombocytopenia. Maced J Med Sci. \\ 2013 Mar15;6(1):50-55 http://dx doi.org/10.3889/ \\ 2013Mar15,6(1):50-55.hte:///dx doi.org/10.3889/ \\ 20120261. \\ Key words: immune thrombocytopenic purpura; \\ monoclonal anti-CD20 antibodies; rituximab. \\ Correspondence: Marica Pavkovic, MD. \\ University Clinic for Hematology, Medical Faculty, \\ ul.Vodnjanska 17, 1000 Skopje, Republic of \\ Macedonia. E-mail:pavkovicm@yahoo.com \\ Received: 30-Apr-2012; Revised: 26-Aug-2012 \\ Accepted: 28-Aug-2012; Online first: 07-Nov-2012 \\ Copyright: (c) 2012 Pavkovic M. This is an open- \\ access article distributed under the terms of the \\ Creative Commons Attribution License, which \\ permits unrestricted use, distribution, and \\ reproduction in any medium, provided the origina \\ author and source are credited. \\ Competing Interests: The author have declared \\ that no competing interests exist.
}

\begin{abstract}
Immune thrombocytopenia (ITP) is an autoimmune disease of unknown etiology, characterized by isolated thrombocytopenia and the absence of any underlying cause for thrombocytopenia. Corticosteroids are the standard first line treatment for patients with symptomatic disease, inducing platelet count recovery in $70-80 \%$ of patients; but in many cases, steroid tapering or withdrawal is followed by a decrease of platelet count and the need for additional treatment. Splenectomy is still the standard salvage therapy in cases refractory to corticosteroid therapy. In the past decade monoclonal anti-CD20 antibodies (Rituximab) are being increasingly used in patients with refractory ITP and other autoimmune diseases. Recent studies show that Rituximab is useful in the treatment of patients with chronic and refractory ITP. We report two cases with chronic ITP treated with standard dose of Rituximab in four weekly doses and than continue to receive maintenance therapy with Rituximab for 2 years.
\end{abstract}

\section{Introduction}

Immune thrombocytopenia or immune thrombocytopenic purpra (ITP) is an autoimmune disease of unknown etiology, characterized by thrombocytopenia due to the presence of platelet autoantibodies specific for platelet membrane glycoproteins, such as GPIIb/IIla, GPIb/IX and GPla/Ila [1]. These autoantibodies cause an increased destruction of autoantibody-opsonized platelets by FcyReceptor bearing phagocytic cells and impaired megakaryocyte maturation with reduced platelet production [2]. The etiology of ITP remains unclear, but both genetic and environmental factors are thought to play role in the development of the disease. ITP in adults is a chronic condition with insidious onset and varying severities of thrombocytopenia, more common in women than in men (2:1). 
According to the recent international consensus report [3], ITP is characterized by isolated thrombocytopenia, defined as platelet count $<100 \times 10^{\%}$ $L$ and the absence of any underlying cause for thrombocytopenia. It can be classified by duration into newly diagnosed, persistent (3-12 month duration) and chronic (>1 year duration). Because severe bleedings are rare in patients with ITP and they occur when platelet counts are $<10 \times 10^{9} / \mathrm{L}$, the main goal of therapy is to maintain a safe platelet count (not necessarily a normal count) to prevent major bleeding and to avoid adverse effects of therapy [4].

Glucocorticosteroids are the standard first line treatmentfor patients with symptomatic disease, inducing platelet count recovery in $70-80 \%$ of patients; but in many cases, steroid tapering or withdrawal is followed by a decrease of platelet count and the need for additional treatment [5]. Splenectomy is still the standard salvage therapy in cases refractory to corticosteroid therapy. About $40 \%$ of splenectomized patients either do not respond or relapse after surgery [6], approximately 10\% of patients have early perioperative or delayed infections, and some develop surgical complications, although rarely fatal. In case of relapse or resistant to corticosteroids ITP, other immunosuppressive drugs can be used including azathioprine, cyclosporine A, cyclophosphamide, danazol, mycophenolate mofetil, all with marginal efficacy [7-11]. Intravenous immunoglobulin (IVIG) and anti-D are also used in the treatment of ITP, especially when rapid improvement of platelet count is needed (in case of life-threatening bleeding or prior to splenectomy or other surgical procedures). However, neither modality induces a long-term remission [12]. Recently, romiplostim and eltrombopag, two new thrombopoetin receptor agonists, have shown potent activity in ITP, but these agents do not act on the underlying disease mechanism, and therapeutic efficacy is dependent on continual administration [13, 14].

Rituximab is a chimeric monoclonal antibody specific for CD20 antigen that is expressed on mature $B$ cells. Rituximab administration cause marked, but transit B-cell depletion, and this effect has been used for the treatment of several autoimmune diseases [15-17]. Rituximab is most commonly used in dose of $375 \mathrm{mg} / \mathrm{m}^{2}$, in four consecutive weekly doses, and it induce overall early response rate in patients with chronic ITP of 40$70 \%$ with complete response rate of 20 to $50 \%[18,19]$. Bhagirath et al. [20] have used Rituximab in chronic relapsing thrombotic thrombocytopenic purpura (TTP) in 4 doses $\left(375 \mathrm{mg} / \mathrm{m}^{2}\right)$ every four months for one year, after initial induction treatment. This agent is consider as safe, although few recent studies reported toxicities, including death (not necessarily attributable to rituximab) in nearly $3 \%$ of cases [18]. Long term therapy with Rituximab is associated with increased risk of infections like hepatitis $\mathrm{B}$ reactivation, herpes zoster reactivation, progressive multifocal leukoencephalopathy, etc. More prospective randomized trials are necessary to evaluate long-term efficacy and safety of Rituximab in the treatment of patients with ITP.

In this paper we report two female patients with chronic steroid-dependent and refractory ITP treated with Rituximab in our institutions. Patients with steroiddependent and refractory ITP represent significant medical problem due to a constant need of treatment and frequent medical controls. Management of these patients refractory to standard treatment with corticosteroids and splenectomy is difficult, because the response rates to all available treatments at the moment is low and are not long-lasting [4].

\section{Case report}

Two female patients with chronic refractory or unresponsive ITP were treated with maintenance therapy with Rituximab in our institution. Both patients met the diagnostic criteria for ITP a) platelet count below $100 \times 10 \%$ $L$ in peripheral blood, b) normal or increased megakaryopoesis on bone marrow examination, and c) the absence of clinically apparent associated conditions or causes of thrombocytopenia like lymphoproliferative disease, chronic liver disease, HIV infection, or other viral or bacterial infections. The medical records of these patients were reviewed for the clinical and laboratory information regarding their diagnosis, initial treatment, splenectomy and after treatment follow-up. The severity of thrombocytopenia was classified into: severe (platelets $<30 \times 10^{9} / \mathrm{L}$ ); moderate (platelet count:30$50 \times 10^{\circ} / \mathrm{L}$ ) and mild (platelets $>50 \times 10^{\circ} / \mathrm{L}$ ).

Rituximab was administered at a dose of $375 \mathrm{mg} /$ $\mathrm{m} 2$ intravenously once weekly for a total of 4 infusions (days 1, 8, 15 and 22) on an outpatient basis. Maintenance therapy was continued every three months for two years at a dose of $375 \mathrm{mg} / \mathrm{m} 2$. Response was evaluated after 4 weeks of treatment. The response criteria to Rituximab treatment were defined as: complete response (CR), a rise of platelet counts $>100 \times 10^{\circ} / \mathrm{L}$; partial response or response (R), a rise of platelet counts $>30 \times 10^{9} / \mathrm{L}$; and no response (NR) when there was no rise in platelet count $>30 \times 10^{\circ} / \mathrm{L}$. 


\section{Case 1}

First case is a female patient born 1974, diagnosed with ITP in 1997 at the age of 23 years. She referred to our Clinic due to isolated thrombocytopenia and skin hemorrhage, without any other symptoms and physical findings. Platelet count at diagnosis was $14 \times 10 \%$ $\mathrm{L}$, with normal hemoglobin level and leukocyte count, and normal peripheral blood smear. Bone marrow analysis showed increased number of megakaryocytes, with otherwise normal hematopoesis. Abdominal ultrasound was normal, as well as all biochemical and serological analysis. Diagnosis of primary immune thrombocytopenia or ITP was made and we started with first-line treatment with prednisone in standard dose of $1 \mathrm{mg} / \mathrm{kg}$. After 1 month platelet count have rose to $83 \times 10 \% / \mathrm{L}$, but started to decrease to $20 \times 10 \% / \mathrm{L}$ with steroid tapering. After 10 months from diagnosis she was splenectomized, due to severe thrombocytopenia resistant to corticosteroid treatment (Figure 1). Three years after splenectomy platelet count was normal and above $150 \times 10^{9} / \mathrm{L}$, then it started to decrease bellow $100 \times 10^{\%} / \mathrm{L}$. Relapse was noticed in March 2001 with drop of platelet count to $7 \times 10^{9} / \mathrm{L}$. Abdominal CT scan was normal (no signs of accessory spleen) and we started with Rituximab treatment at a dose of $375 \mathrm{mg} / \mathrm{m} 2$ in four weekly doses. One month after the $4^{\text {th }}$ dose of Rituximab platelet count rose to maximum of $97 \times 10^{9} / \mathrm{L}$ and partial response was achieved. We continued with maintenance therapy with Rituximab at the same dose, every three months for two years (total of 8 doses). During those two years platelet count was between 50 and $100 \times 10^{9} / \mathrm{L}$. At the end of this period drop in a platelet count to $3 \times 10^{9} / \mathrm{L}$ was registered and we started with prednisone at low doses of $25 \mathrm{mg} /$ day. Two months after prednisone was initiated, platelet count has risen to $93 \times 10^{9} / \mathrm{L}$. Corticosteroid treatment last for 18 months and it was stopped due to stable platelet count between $50-100 \times 10^{9} / \mathrm{L}$ and no hemorrhagic symptoms. Since the beginning of year 2006 patient is without therapy, with platelet count between $50-100 \times 10^{9} / \mathrm{L}$ and no symptoms. Last control was in December 2010 when platelet count was $137 \times 10^{9} / \mathrm{L}$ without any treatment (Figure 1 ). In this

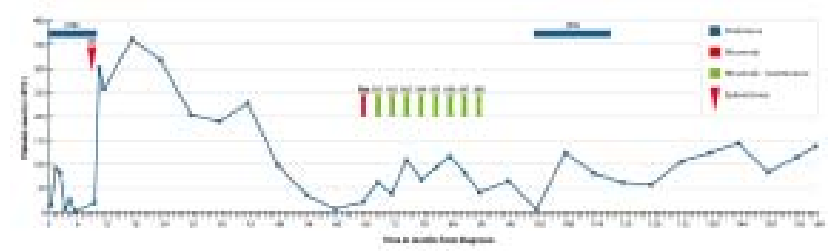

Figure 1: Response to different treatments in Patient No.1. case, Rituximab induced partial response that last during the treatment and relapse of ITP was diagnosed just when maintenance therapy was finished. The benefit of treatment with Rituximab was no need of corticosteroid treatment for more than 2 years.

\section{Case 2}

Second case is also a female patient born 1969 , diagnosed with ITP in 1980 at the age of 11 years and she was treated at Pediatric Department with corticosteroids for 6 months with good initial response. She had two successful pregnancies in 1988 and 1992 without any treatment for ITP. She referred to our Clinic for the first time in 1996, due to isolated mild thrombocytopenia, without any symptoms and physical findings (Figure 2). Platelet count at diagnosis was $56 \times 10^{9} / \mathrm{L}$, with normal hemoglobin level and leukocyte count, and normal peripheral blood smear. After 6 months of follow up without treatment, platelet count dropped to $6 \times 10^{9} / \mathrm{L}$ and she complained to prolonged menstrual bleeding. Bone marrow analysis was done and showed increased number of megakaryocytes, with otherwise normal hematopoesis. Abdominal ultrasound was normal, as well as all biochemical and serological analysis. We started with first-line treatment with prednisone at standard dose of $1 \mathrm{mg} / \mathrm{kg}$. Response to corticosteroids was slow and partial, with raise in platelet count to maximum of $77 \times 10^{9} / \mathrm{L}$. Every time when we try to decrease or stop prednisone, platelet count started to drop to values bellow 20x10\%/L. Patient was dependent to corticosteroid treatment with platelet count constantly below $20 \times 10^{9} / \mathrm{L}$ and hemorrhagic symptoms like genital bleeding, epistaxis, and skin purpura. She also had complication in a form of herpes virus infection due to immunosuppressive therapy with corticosteroids. She refused to proceed with splenectomy. We have performed second examination of bone marrow with the same findings of increased megakaryopoesis. In January 2003, we started Rituximab treatment at dose of $375 \mathrm{mg} / \mathrm{m} 2$ in four weekly doses (Figure 2). One month after the last dose of Rituximab platelet count rose to maximum of $94 \times 10^{\%} / \mathrm{L}$ and partial response was achieved. We continued with maintenance therapy with Rituximab at the same dose, every three months for two years (total of 8 doses). During those two years platelet count was between 50 and $150 \times 10^{9} / \mathrm{L}$. She had partial response to Rituximab and one year after the last dose of Rituximab she was without any therapy, with platelet count between 50 and 100x10\%/L. In September 2005, drop in platelet count to $3 \times 10^{9} / \mathrm{L}$ was noticed and prednisone treatment was started again. Partial response was achieved. In the 


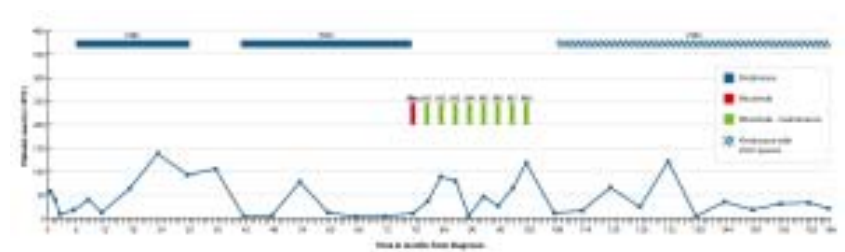

Figure 2: Response to different treatments in Patient No.2.

period of 2006-2010 she was dependent on corticosteroids with platelet count between $50-100 \times 10^{9}$ L. Every time when we stopped prednisone, marked drop in platelet count to $2-3 \times 10^{9} / \mathrm{L}$ was noticed. Last control was in December 2010 when platelet count was $22 \times 10^{9} / \mathrm{L}$ with low dose of prednisone (10 mg per day). In this case also, Rituximab induced only partial response that last during the treatment and relapse of thrombocytopenia was diagnosed 1 year after maintenance therapy was finished. The benefit of treatment with Rituximab was no need of corticosteroid treatment for more than 3 years.

\section{Discussion}

Management of patients with ITP refractory to standard treatment with corticosteroids and splenectomy is difficult, because the response rates to all available treatments at the moment are low and are not longlasting. In the past decade monoclonal anti-CD20 antibodies (Rituximab) are being increasingly used in patients with refractory ITP and other autoimmune diseases. There are many studies reporting the results of Rituximab therapy in patients with ITP. Arnold et al [18] systematically reviewed literature and referred overall response rate of $62.5 \%$ with significant toxicities, including dead in $2.9 \%$ of cases. Stasi et al. [21] showed that 10 of 25 patients with chronic and refractory ITP, already treated with 2 to 5 lines of treatment, respond to Rituximab and this response was maintained for 6 months or longer in $28 \%$ of patients. Results from this study indicate that rituximab therapy has a limited but valuable effect in patients with chronic ITP. Due to its mild toxicity and the lack of effective alternative treatments for patients with refractory ITP, Rituximab should be considered in patients who do not respond to first and second line of treatment.

Garcia-Chaveez et al. [22] reported a $67 \%$ sustained response in 18 pretreated ITP patients, while Godeau et al. [23] reported promising 1-year response in $40 \%$ of patients. Aleem et al. [24] retrospectively studied 24 patients with refractory ITP, treated with
Rituximab and $34 \%$ of patient's sustained response after 6 months, 24\% responses sustained after 1 year.

Zaja et al. [25] conducted the first randomized clinical trial in which they have compared the efficacy of treatment with Rituximab+Dexamethasone and Dexamethasone alone, in 103 newly diagnosed or chronic patients with ITP that have not received any treatment before (treatment-naï ve patients).

Arnold et al. [26] performed a pilot randomized trial to determine the feasibility of recruitment, protocol adherence, and blinding of a larger trial of rituximab versus placebo; and to evaluate the potential efficacy of adjuvant rituximab in ITP. Nonsplenectomized adults with newly diagnosed or relapsed ITP who were receiving standard ITP therapy for a platelet count below $30 \times$ 10(9)/L were randomly allocated to receive 4 weekly infusions of $375 \mathrm{mg} / \mathrm{m}^{2}$ rituximab or saline placebo. Sixty patients were recruited over 46 months, which was slower than anticipated. After 6 months, there was no difference between rituximab and placebo groups for the composite outcome of any platelet count below $50 \times$ 10(9)/L, significant bleeding or rescue treatment once standard treatment was stopped

Results from this study indicate that the combination of Dexamethason and Rituximab improves patient outcomes without compromising the safety. The relatively sustained duration of response in some patients suggests a beneficial effect and this treatment gives an option for second-line therapy, particularly in patients not responding to steroids and as an alternative to splenectomy.

Long term therapy with Rituximab may have potentially harmful effects, including the risk of hepatitis $B$ reactivation, herpes zoster reactivation, progressive multifocal leukoencephalopathy, etc. These long-term side effects are well known and described in lymphoma patients treated with rituximab $[27,28]$ but fortunately there were rare in patients with ITP treated with monoclonal anti-CD20 antibodies [18]. Most common adverse events of Rituximab treatment in ITP patients were infusional reactions and infections like pneumonia and meningitis [18].

Our results from two patients with refractory ITP were similar to already repotted results from other studies. Previous studies didn't use maintenance therapy, only four weekly doses of Rituximab. We used maintenance therapy with Rituximab and we report our experience with it. Both patients had partial response to Rituximab treatment and response sustained for short period (1 
year in second case, and only during the maintenance therapy in the first case). We didn't observe any side effects from Rituximab treatment and the benefit from it was no need of other therapy and unwanted side effects from it (ex. steroids, cyclophosphamide, or other immunosuppressive agents).

In conclusion, Rituximab is well tolerated agent and could be useful in the treatment of some patients with refractory or unresponsive ITP, despite the relatively low response rate and short sustained remissions.

\section{References}

1. McMillan R. Chronic idiopathic thrombocytopenic purpura. N Eng J Med. 1981;304: 1135-1147.

2. Cines DB, Blanchette VS. Immune thrombocytopenic purpura. N Eng J Med. 2002;346: 995-1008.

3. Rodeghiero F, Stasi R, Gernsheimer T, et al. Standardization of terminology, definitions and outcome criteria in immune thrombocytopenic purpura of adults and children: report from an international working group. Blood. 2009; 113 :2386-2393.

4. Portielje Je, Westendorp RG, Kluin-Nelmand HC, Brand A. Morbidity and mortality in adults with idiopathic thrombocytopenic purpura. Blood. 2001;97: 2549-54.

5. George JN, Woolf SH, Raskob GE, et al. Idiopathic thrombocytopenic purpura: a practice guideline developed by explicit methods for the American Society of Hematology. Blood. 1996;88:3-40.

6. Kojouri K, Vesely SK, Terrell DR, George JN. Splenectomy for adult patients with idiopathic thrombocytopenic purpura: a systemic review to assess long-term platelet count responses, prediction of response, and surgical complication. Blood. 2004;104: 2623-2634.

7. Quiquandron I, Fenaux P, Caulier MT, et al. Re-evaluation of the role of azathiprine in the treatment of adult chronic idiopathic thrombocytopenic purpura: a report of 53 cases. $\mathrm{Br}$ J Haematol. 1990;74: 223-228.

8. Kappers-Klunne MC, van't Veer MB. Cyclosporine A for the treatment of patients with chronic idiopathic thrombocytopenic purpura refractory to corticosteroids or splenectomy. $\mathrm{Br} \mathrm{J}$ Haematol. 2001;114: 121-125.

9. Verlin M, Laros RKJr, Penner JA. Treatment of refractory thrombocytopenic purpura with cyclophosphamide. Am J Hematol. 1976;1: 97-104.

10. Maloisel F, Andres E, Zimmer J, et al Danazol therapy in patients with chronic idiopathic thrombocytopenic purpura: Iong term results. Br J Haematol. 2004;116: 590-594.

11. Provan D, Moss AJ, Newland AC, Bussel JB. Efficacy of mycophenolate mofetil as a single agent therapy for refractory immune thrombocytopenic purpura. Am J Hematol. 2006;81:19-25.

12. Blanchette VS, Luke B, Andrew M, et al. A prospective, randomized trial of high dose intravenous immune globulin $G$ therapy, oral prednisone therapy, and no therapy in childhood acute immune thrombocytopenic purpura. J Pediatr. 1993;123: 989-995.

13. Kuter DJ, Bussel JB, Lyons RM et al. Efficacy of romiplostim in patients with chronic immune thrombocytopenic purpura: a double-blind randomized controlled trial. Lancet. 2008; 371: 395-403.

14. Bussel JB, Cheng G, Saleh MN, et al. Eltrombopag for the treatment of chronic idiopathic thrombocytopenic purpura. N Engl J Med. 2007;357: 2237-2247.

15. Edwards JC, Szczepanski L, Szechinski J, et al. Efficacy of B-cell-targeted therapy with rituximab in patients with rheumatoid arthritis. N Engl J Med. 2004;350: 2572-2581.

16. Ahmed ER, Spigelman Z, Cavacini LA, Posner MR. Treatment of pemphigus vulgaris with rituximab and intravenous immune globulin. N Engl J Med. 2006;355: 17721779.

17. Zecca M, Nobili B, Ramenghi U, et al. Rituximab for the treatment of refractory autoimmune hemolytic anemia in children. Blood. 2004;103: 2925-2928.

18. Arnold DM, Dentali F, Crowther MA, et al. Systematic review: efficacy and safety of rituximab for adults with idiopathic thrombocytopenic purpura. Ann Intern Med. 2007;146: 25-33.

19. Zaja F, Baccarani M, Mazza P, et al. Dexamethason plus rituximab yields higher sustained response rates than dexamethasone monotherapy in adults with primary immune thrombocytopenia. Blood. 2010;115: 2755-2762.

20. Bhagirath VC, Kelton JG, Moore J, Arnold DM. Rituximab maintenance for relapsed refractory thrombotic thrombocytopenic purpura. Transfusion. 2012 Apr 4. doi: 10.1111/j.1537-2995.2012.03635.x.

21. Stasi R, Pagano A, Stipa E, Amadori S. Rituximab chimeric anti-CD20 monoclonal antibody treatment for adults with chronic idiopathic thrombocytopenic purpura. Blood. 2001;98: 952-957.

22. Garcia-Chavez J, Majluf-Cruz A, Montiel-Cervantes L, et al. Rituximab therapy for chronic and refractory immune thrombocytopenic purpura: long-term follow-up analysis. Ann Hematol. 2007;86: 871-877.

23. Godeau B, Porcher R, Fain O, et al. Rituximab efficacy and safety in adult splenectomy candidates with chronic immune thrombocytopenic purpura: results of a prospective multicenter phase 2 studies. Blood. 2008;112: 999-1004.

24. Aleem A, Alaskar AS, Algahtani F, et al. Rituximab in 
immune thrombocytopenia: transit responses, low rate of sustained remissions and poor response to further therapy in refractory patients. In J Hematol. 2010;92: 283-288.

25. Zaja F, Baccarani M, Mazza P, Bocchia M, et al. Dexamethasone plus rituximab yields higher sustained response rates than dexamethasone monotherapy in adults with primary immune thrombocytopenia. Blood. 2010; 115: 2755-2762.

26. Arnold DM, Hedde NM, Carruthers J, et al. A pilot randomized trial of adjuvant rituximab or placebo for nonsplenectomized patients with immune thrombocytopenia. Blood. 2012;119:1356-62.

27. Tsutsumi Y, Kawamura T, Saitoh S et al. Hepatitis B reactivation in a case of Non-Hodgkin's lymphoma treated with chemotherapy and rituximab: necessity of prophylaxis for hepatitis $B$ virus reactivation in rituximab therapy. Leukemia Lymphoma. 2004;45: 627-629.

28. Yang SH, Kuo SH. Reactivation of hepatitis B virus during rituximab treatment of patient with follicular lymphoma. Ann Hematol. 2008;87: 325-327. 